\title{
Navigating the Fog: A Correlated XRM and FIB-SEM Imaging Pipeline for the Rapid and Precise Spatial Targeting of Rare Structures in Biological Samples
}

\author{
Matthew S. Joens ${ }^{1}$, Daniel J. Geanon ${ }^{1}$, Peter O. Bayguinov ${ }^{1}$, Kory J. Lavine ${ }^{2}$, James A.J. Fitzpatrick ${ }^{1,3,4}$ \\ ${ }^{1 .}$ Center for Cellular Imaging, Washington University School of Medicine, Saint Louis, USA. \\ 2. Department of Medicine, Cardiovascular Division, Washington University School of Medicine, Saint \\ Louis, USA. \\ 3. Departments of Cell Biology \& Physiology and Neuroscience, Washington University School of \\ Medicine, Saint Louis, USA \\ 4. Department of Biomedical Engineering, Washington University, Saint Louis, USA.
}

Bridging the gap between microscopic and nanoscopic length scales has long since been an obstacle in microscopy. Although much effort has gone into the development of robust methodologies for correlating data obtained using both light and electron microscopies, there still exists an inherent incompatibility between sample preparations that are used for each technique [1]. The heavy metal stains that are used routinely in electron microscopy often render the sample completely opaque [2], which leads to fundamental issues in successfully targeting a given spatial region in a sample. Alternatively, X-ray microscopy (XRM) has the ability to image through an entire resin block at highresolution and in a non-destructive manner. Since the contrast agents used for 3D electron microscopy also attenuate $\mathrm{x}$-ray transmission, the resulting tomogram can be analyzed to identify rare structures or specific regions of interest (ROI) in relation to the orthogonal plane that correlates to the top of the block face. By measuring the axial (z-distance) between these two points, excess resin and sample can be rapidly and precisely trimmed to the exact depth of the ROI, perfectly exposing the target region for FIB-SEM 3D nanotomography. The resulting data can then be combined to contextually traverse large length scales from the whole organism down to the spatial length of a single cell.

To demonstrate the utility of this technique, we have focused on a zebrafish model of dilated cardiomyopathy, where the embryonic heart muscle is often shrunken, displaced, and nearly impossible to identify by optical means after heavy metal staining. Zebrafish were prepared by means of an OTO preparation for three-dimensional analysis and flat embedded in plastic resin [3]. The resin block was scanned in an X-ray Microscope (Zeiss Xradia Versa 520, Pleasanton, CA) initially using the 4x scintillated objective to obtain an overview tomogram of the sample and subsequently with the $20 \mathrm{x}$ scintillated objective to analyze the cardiac sac in greater detail. Once a ROI was selected, the two XRM datasets were digitally overlaid with one another and the precise distance from the ROI to the desired orthogonal plane representing the block surface was measured (Fig. 1A). This material was then removed by means of the auto trim function of an ultramicrotome or a rapid miller/grinder. The removal of this material seems to be accurate to $+/-10 \mu \mathrm{m}$. Once this layer was removed, the block was mounted on an aluminum stub where the polished face that is visualized in the FIB-SEM reflects that of the XY orthogonal plane in the XRM tomogram (Fig. 1B). Since FIB-SEM is capable of milling a trench anywhere on the block face, correlating the $\mathrm{XZ}$ and $\mathrm{YZ}$ orthogonal planes is unnecessary in this case. Utilizing the ATLAS5 correlative workspace (Fibics, Ottawa, Canada), XY orthogonal slices from the XRM tomogram were overlaid and aligned with common features that were visible in both SEM and FIB imaging modes (Fig. 1C). Once aligned, the ATLAS5 workspace allows the designation of an ROI for a nanotomography data acquisition run at an exact spatial location. Depending on the resolution needed to identify structures in the XRM, this entire workflow can take as little as 4 hours. 


\section{References:}

[1] Lucas, M. S., et al., Method Cell Biol 111 (2012), p. 325.

[2] Peddie, C. J., and Collinson, L. M., Micron 61 (2014), p. 9.

[3] Deerinck, T., et al., Microscopy and Microanalysis 16 (SupplementS2) (2010), p. 1138.
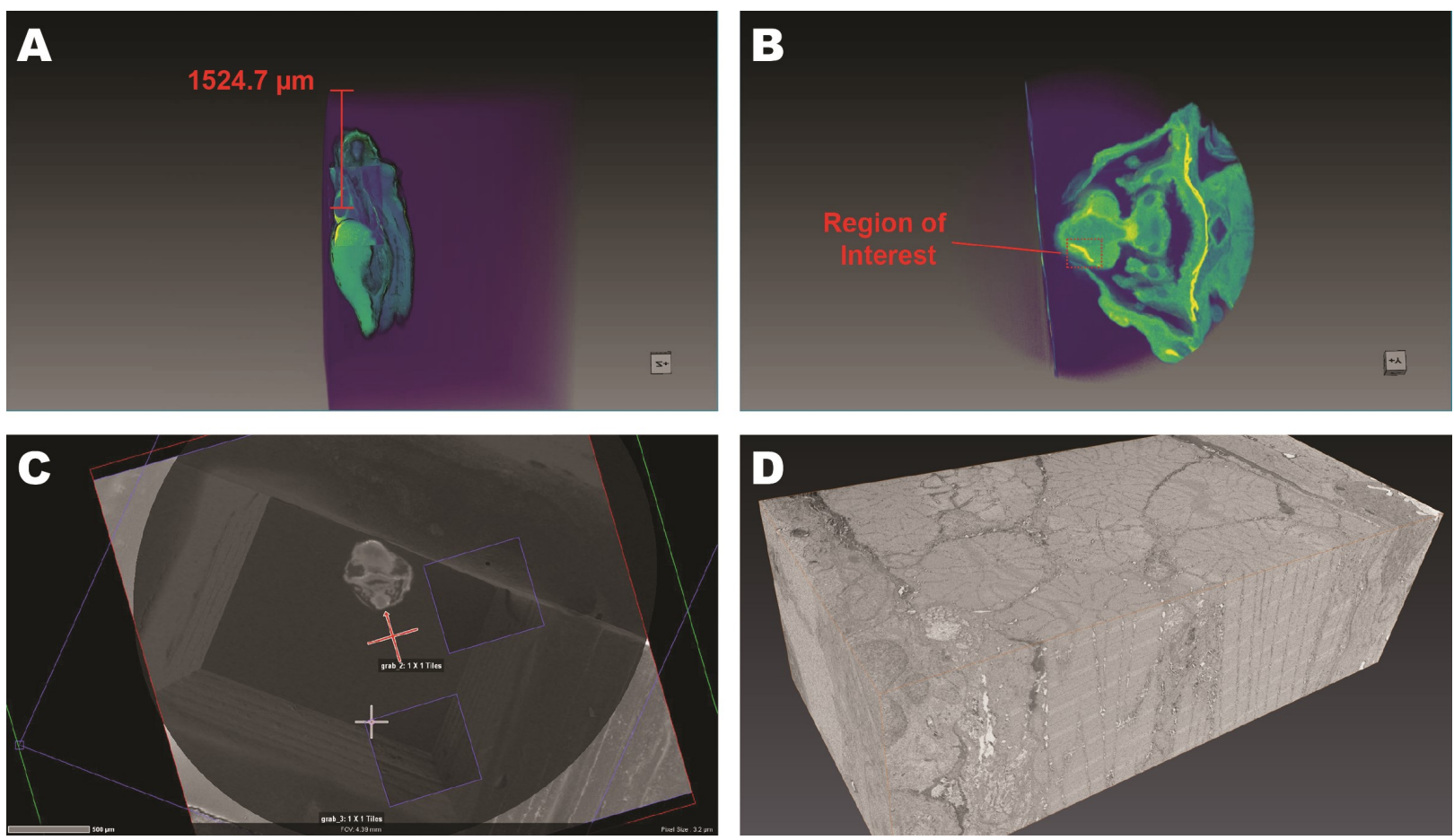

Figure 1. Correlative XRM/FIB-SEM workflow. A) Overlaid 4x and 20x XRM datasets are used to measure bulk plastic removal. B) XY plane in XRM is used to target ROI. C) ATLAS workflow to align XRM, FIB, and SEM images. D) Resulting FIB-SEM volume of ROI 